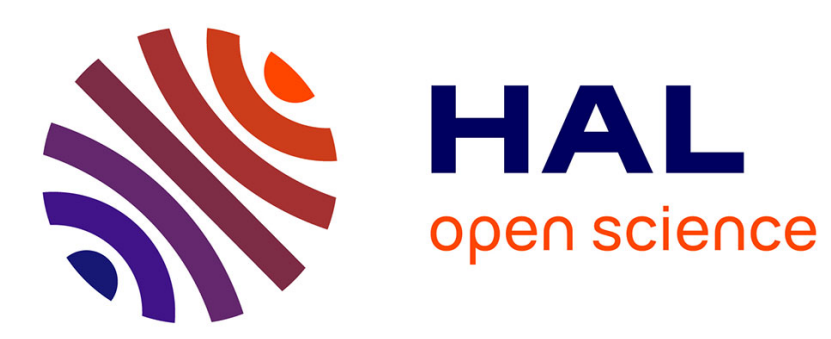

\title{
A Model Electron Transfer Reaction in Confined Aqueous Solution
}

Jean-François Olivieri, Damien Laage, James T Hynes

\section{To cite this version:}

Jean-François Olivieri, Damien Laage, James T Hynes. A Model Electron Transfer Reaction in Confined Aqueous Solution. ChemPhysChem, 2021, 10.1002/cphc.202100351 . hal-03369284

\section{HAL Id: hal-03369284 \\ https://hal.sorbonne-universite.fr/hal-03369284}

Submitted on 7 Oct 2021

HAL is a multi-disciplinary open access archive for the deposit and dissemination of scientific research documents, whether they are published or not. The documents may come from teaching and research institutions in France or abroad, or from public or private research centers.
L'archive ouverte pluridisciplinaire HAL, est destinée au dépôt et à la diffusion de documents scientifiques de niveau recherche, publiés ou non, émanant des établissements d'enseignement et de recherche français ou étrangers, des laboratoires publics ou privés. 


\title{
A Model Electron Transfer Reaction in Confined Aqueous Solution
}

\author{
Jean-François Olivieri, ${ }^{[a]}$ Damien Laage ${ }^{\star[a]}$ and James T. Hynes ${ }^{\star[a, b]}$
}

[a] J.-F. Olivieri, Dr. D. Laage, Prof. J.T. Hynes

PASTEUR, Department of Chemistry, École Normale Supérieure, PSL University, Sorbonne Université, CNRS

75005 Paris, France

E-mail: damien.laage@ens.psl.eu

[b] Prof. J.T. Hynes

Department of Chemistry, University of Colorado

Boulder, CO 80309, USA

E-mail: casey.hynes@ens.psl.eu

\begin{abstract}
Liquid water confined within nanometer-sized channels exhibits a strongly reduced local dielectric constant perpendicularly to the wall, especially at the interface, and this has been suggested to induce faster electron transfer kinetics at the interface than in the bulk. We study a model electron transfer reaction in aqueous solution confined between graphene sheets with classical molecular dynamics We show that the solvent reorganization energy is reduced at the interface compared to the bulk, which explains the larger rate constant However, this facilitated solvent reorganization is due to the partial desolvation by the graphene sheet of the ions involved in the electron transfer and not to a local dielectric constant reduction effect.
\end{abstract}

\section{Introduction}

The impact of confinement on structural and dynamical properties of liquids is of interest in a broad variety of contexts, including, e.g. transport within nanofluidic devices to catalysis of chemical reactions in nanoreactors ${ }^{[1-5]}$. In one of these, recent experimental ${ }^{[6,7]}$ and simulation ${ }^{[8-12]}$ studies have suggested that the dielectric constant of water is dramatically reduced in nanometer-size channels along the direction perpendicular to the channel wall, and exhibits very strong anisotropy with respect to the confining media. Such effects could have considerable importance for charge transfer reactions: such reduced permittivity could facilitate transfer by reducing solvent reorganization penalties which slow such reactions. Indeed, it has been suggested that very rapid electron transfer (ET) reaction rates at interfaces determined in recent experimental measurements could be due to the reduced interfacial dielectric constant. ${ }^{[13]}$

In the present work, we explore such a possibility for key aspects of a model ET reaction ${ }^{27}$ in water bounded by (here uncharged) graphene sheets, whose separation is fixed, a system of considerable interest in a wide variety of arenas. ${ }^{[14-17]}$ Our study employs both molecular dynamics simulations and the theoretical results of previous efforts ${ }^{[12,18,19]}$ on water confined between such sheets, in particular a recent investigation ${ }^{[12]}$ of water's dielectric constant behavior in such an environment.

Many prior simulation studies (see e.g. ${ }^{[20-32]}$ ) have focused on ET between a redox species in solution and a metallic electrode. These have identified that for these systems, the major effect on ET reaction is due to the interface's metallic nature. The image charge effect due to electronic polarization in the metal reduces the solvent reorganization energy $\lambda^{[28,32]}$, accelerating the ET reaction, an effect which can extend several $\mathrm{nm}$ from the interface [28]. In addition, $\lambda$ may be influenced by modified interfacial solvation shell structure. ${ }^{[21,26,30,33]}$

Here we are instead concerned with an aqueous solution model ET reaction confined between graphene surfaces, which do not involve such significant image charge effects; this allows a focus of attention on interfacial region (and beyond) solvation effects, including those related to water's dielectric constant behavior. In this first effort, we focus on an ET reaction not involving charge transfer to or from the graphene, but rather examine an ET for a solute within the water solvent. Further, we do not address all aspects of the ET reaction rate, concentrating instead on the reorganization energy $\lambda$ which has an important impact on that rate $^{[34-37]}$. The model employed ${ }^{[38]}$ is quite simple and, as discussed within, has a number of simplifying features which allow this focus on solvation effects unencumbered by other important, but also obscuring, features influencing the reaction. In this fashion we are able to elucidate the role of several features including the water dielectric constant - governing the behavior of $\lambda$ both close to and away from the graphene surfaces. In particular, we show that the reduced solvent reorganization energy for ET at the graphene interface is not due to the reduced dielectric constant component perpendicular to the wall. Instead, we show that the small reorganization energy results from the partial desolvation induced by the wall for solutes lying in the first layer. The outline of the remainder of this paper is the following. We first describe the model electron transfer reaction and introduce the key activation free energy and solvent reorganization energy quantities, followed by a presentation of our simulation methodology. We then analyze the free energy curves and reorganization energies obtained from our simulations in different geometries for the solute within the confined water slab, with a special focus on the role of the effective dielectric constant. Finally, we offer some concluding remarks.

\section{Model electron transfer reaction}

The model ET reaction system in graphene plate-confined water selected for this molecular dynamics study is closely related to that introduced by Zichi et al ${ }^{[38]}$. It involves two atomic charge centers $\mathrm{A}$ and $\mathrm{B}$ separated by a variable distance $R_{A B}$, the reaction consisting of the single electron transfer

$$
\mathrm{A}^{-1 / 2} \mathrm{~B}^{+1 / 2} \rightarrow \mathrm{A}^{+1 / 2} \quad \mathrm{~B}^{-1 / 2}
$$

This ET has the advantage that it is thermodynamically symmetric, i.e., the reaction free energy $\Delta G_{\mathrm{rxn}}=0$, in the bulk. This both 
avoids any influence of the reaction thermodynamics on the ET barrier in the bulk and, most especially, allows a clear exposure of any reaction asymmetry which may be introduced in the interfacial region by the presence of the graphene surfaces.

In contrast to Ref. ${ }^{[38]}$, we do not address in our study the electronic coupling $V_{e l}$ between the diabatic reactant and product valence bond states, which would (strongly) vary with the A-B separation $R_{A B}$ and importantly impact the ET reaction rate: lowering the barrier in the adiabatic regime (smaller $R_{A B}$, larger $\left.V_{e l}\right)$ and providing a rate prefactor in the nonadiabatic tunneling regime (larger $R_{A B}$, smaller $V_{e l}$ ). Omitting these impacts allows an exclusive focus on the solvent reorganization energy $\lambda$, which importantly contributes to the rate whatever the value of $V_{e l}$ (cf eq. 2).

On the other hand, in order to clarify the significance of key quantities that we calculate, it is useful to quote the well-known Marcus equation for a nonadiabatic, small $V_{e l}$ ET reaction barrier $\Delta G^{\ddagger}$ involving the already mentioned reorganization energy and the reaction free energy ${ }^{[34-37]}$

$$
\Delta G^{\ddagger}=\left(\lambda+\Delta G_{r x n}\right)^{2} / 4 \lambda
$$

which shows that the reaction barrier is $\lambda / 4$ for the thermodynamically symmetric reaction. For a symmetric adiabatic reaction, this barrier would be reduced by $V_{\text {el. }}[35,36,39]$ Equation 2 relies on a certain harmonic approximation discussed in a subsequent section, where relevant free energy curves are found via simulation rather than harmonic approximation. Also useful for perspective in our efforts is (in a form appropriate for our model) the Marcus model dielectric continuum approximation for $\lambda^{[34-36]}$

$$
\lambda(R)=\Delta q^{2}(1-1 / \varepsilon)\left(1 / 2 r_{A}+1 / 2 r_{B}-1 / R_{A B}\right)
$$

where $\Delta q=1 \mathrm{e}$ is the transferred charge, $\varepsilon$ is the solvent dielectric constant (with electronic polarization neglected), $r_{A, B}$ are the radii of the $\mathrm{A}$ and $\mathrm{B}$ atoms, and $R_{A B}$ is the $\mathrm{A}-\mathrm{B}$ separation. Equation 3 reflects the generally expected trends in a bulk solution symmetric reaction: $\lambda$ - and thus the ET barrier - decreases as $\varepsilon$ decreases towards 1 , and increases to an asymptotic value as the A-B separation $R_{A B}$ increases. (A feature that will be important for our analysis is that this very large $R_{A B}$ asymptotic limit shares the same dependence on $\varepsilon$ and on $r_{A, B}$ as the familiar Born model ${ }^{[40]}$ solvation free energies $\Delta G_{\text {solv }}{ }^{\text {Born }}$ for $A$ and B: e.g., eq. 3 reduces to the sum of these free energies, scaled by $\left(\Delta q / q_{A}, B\right)^{2}$; in addition, eq. 3 assumes an isotropic environment while this is not the case at the interface, and we will return to this point in the following) The eq. 1 model's van der Waals radii are identical for $A$ and $B$, but the more relevant radii for eq. 3 are the cavity radii, which can be approximated by the location of the first peak in the solutesolvent radial distribution function. This peak occurs at a shorter distance for the anion than for the cation (see Fig. 1f), a wellknown asymmetry for water solvent ${ }^{[41-43]}$. Thus, in the bulk the ET reaction is symmetric, although the solvation free energies for the reaction complex's two charged moieties differ.

In the simulations, several different geometries will be considered (Scheme 1): i) $A$ is within the $1^{\text {st }}$ layer next to graphene and $B$ is located such that the $A B$ vector is normal (perpendicular) to the wall; ii) Both $A$ and $B$ are located within the $1^{\text {st }}$ layer next to graphene, such that the $A B$ vector is parallel to the wall; for both geometries, the A-B separation $R_{A B}$ is varied, with special attention paid to the reference situations (iii) and (iv), in which the perpendicular and parallel geometries are also examined at the midpoint of the water slab. Finally, the A-B system is separately considered in the bulk, for reference.

\section{Methodology}

Classical molecular dynamics simulations are performed with the model ET reactive solute (described above) immersed in SPC/E water within a $6 \mathrm{~nm}$-thick periodic slab between graphene sheets, as well as in a bulk SPC/E water reference system. The two 240atom rigid graphene sheets are constructed from an ideal hexagonal lattice with a $1.42 \AA$ distance between neighboring carbon atoms. There is zero charge placed on the graphene carbon atoms (see Refs. ${ }^{[18,19]}$ for the distinct situation of graphene electrodes held at constant potential and the impact on interfacial water layer structure and dynamics). The simulation box dimensions are $24.58 \times 25.56 \times 62 \AA^{3}$ and the number of water molecules (1150) is determined such that the water has the correct bulk density in the middle of the slab. The Lennard-Jones parameters for sites $A$ and $B$ are $\sigma_{A, B}=2.5 \AA$ and $\varepsilon_{A, B}=100 \mathrm{k}_{B} T$ (additional results for the larger solute parameter $\sigma_{A, B}=3.5 \AA$ are reported in the SI). Graphene carbon Lennard-Jones parameters are taken from ref. ${ }^{[44]}$ where they were optimized to reproduce DFT-based molecular dynamics simulation results (see SI for details). Water molecules are held rigid with the SHAKE algorithm. The Yeh-Berkowitz slab correction ${ }^{[45]}$ to Ewald sums for $2 \mathrm{D}$ systems is employed.

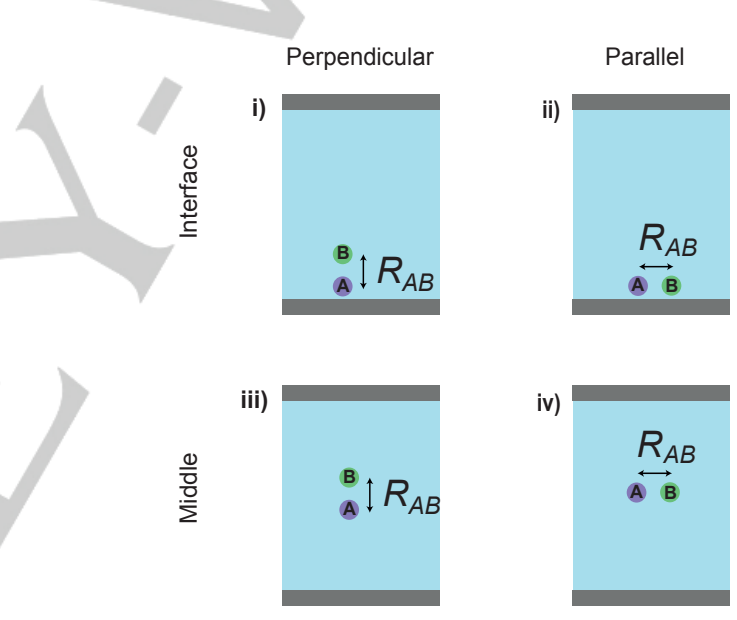

Scheme 1. Different geometries for the A-B pair within the water slab between the graphene plates.

For each system, five independent initial configurations are prepared. Molecular dynamics simulations are run with LAMMPS [46]. After $1 \mathrm{~ns}$ equilibration at $300 \mathrm{~K}, 1 \mathrm{~ns}$ production runs are propagated with a 2 fs timestep and the velocity Verlet integrator in the canonical ensemble, using the velocity rescale thermostat [47] with a 2 ps relaxation time. Configurations are output with a $50 \mathrm{fs}$ interval. Solute-solvent interaction energies are calculated with both reactant and product charge distributions at every output step. This procedure is repeated for each geometry shown in Scheme 1 and for a series of A-B separations by freezing the positions of $A$ and $B$.

The bulk reference system trajectories were produced following the same procedure, with 40 independent starting configurations, using a $30 \times 30 \times 30 \AA^{3}$ box containing 898 water molecules and a pair of $A$ and $B$ solutes whose positions are not constrained.

The free energy profiles of the reactant $(R)$ and product $(P)$ diabatic states are calculated from a series of independent 
trajectories propagated with the mapping potential $V_{\eta}=(1-\eta) V_{R}+$ $\eta V_{P}$, with $\eta$ ranging from 0 (pure state $R$ ) to 1 (pure state $P$ ). The mapping potential is used in this work to generate solvent configurations equilibrated to different electronic structures of the $A B$ pair. The free energy of each diabatic state is reconstructed using the weighted histogram analysis method (wham) ${ }^{[48]}$. While wham is usually employed with harmonic biasing potentials, it can be easily generalized to the present mapping potential simulations by defining the biasing potential as $w_{\eta}^{R}(\Delta E)=V_{\eta}(\Delta E)$ $-V_{R}(\Delta E)=-\eta \Delta E$, and $w_{\eta}^{P}(\Delta E)=(1-\eta) \Delta E$. (Here $\Delta E$ is a solvent coordinate defined at the beginning of the following section.) The diabatic free energy profiles are determined using 11 independent 1 ns-long windows regularly spaced along $\eta$.

The solvation free energy profile of solutes $A$ and $B$ at varied separations from the wall is calculated using a series of independent trajectories where a single solute is present in the box and its distance $z$ to the wall is harmonically constrained by $k$ $\left(z-z_{0}\right)^{2}$ with $k=50 \mathrm{k}_{\mathrm{B}} T / \AA^{2}$ and $z_{0}$ ranges from $1.5 \AA$ to $30 \AA$ in 0.125 $\AA$ increments. Five independent 200 ps trajectories are used for each $z_{0}$ distance and the free energy profile is reconstructed with the wham ${ }^{[48]}$ algorithm.

\section{ET Free Energy Curves}

\section{Reaction Coordinate}

We need to specify a reaction coordinate in order to generate diabatic free energy curves for the ET reaction to determine the reorganization energy $\lambda$ as well as the reaction free energy $\Delta G_{r \times n}$. Here we select a reaction coordinate $\Delta E$ is designed to allow the sampling of different, usually nonequilibrium, arrangements of the reaction solute's environment.

$\Delta E$ is defined as a certain difference in the potential energy of interaction of the different charge states of the reaction solute complex A-B with the water molecules and the graphene plates (eq. 4). We now anticipate that in our model - as to be explained presently - the potential energies in eq. 4 are simply the Coulomb potential interaction between the charges in the reaction solute sites and those of the water solvent molecules. Then

$$
\Delta E(\mathbf{x})=V_{R}(\boldsymbol{x})-V_{P}(\boldsymbol{x})
$$

is the difference of the Coulomb interaction energies of the solvent water molecules with the different varied charge distributions of the reactant $\mathrm{R}$ complex $V_{R}(\mathbf{x})$ and of the product $\mathrm{P}$ complex $V_{P}(\mathbf{x})$, where $\mathbf{x}$ designates the solute and solvent positions. The detailed description of the calculation of the free energy curves in this coordinate is given in the Methodology section.

We now return to the postponed issue of eq. 4's important simplification that only Coulomb reaction complex-water molecule interactions are involved. This results from our reaction model and our restriction to uncharged graphene plates: the nonCoulombic interactions of the model's A and B units with the solvent waters and the graphene plates remain the same independently of the charges on them, and thus completely vanish in the difference eq. 4 .

\section{Free energy curves}

Figure 1 shows the diabatic free energy curves calculated from our simulations in a series of geometries for the $A B$ pair (see Scheme 1) and for $R_{A B}$ distances of $5 \AA$ (which approximately corresponds to a solvent separated ion pair arrangement, see Fig. 1f) and $10 \AA$ (beyond which the electronic coupling exponential decay with the separation will make the ET reaction very slow). In the following, we analyze these free energy curves and their harmonic approximations, and successively discuss the reaction free energy, reaction free energy barrier and solvent reorganization energy.
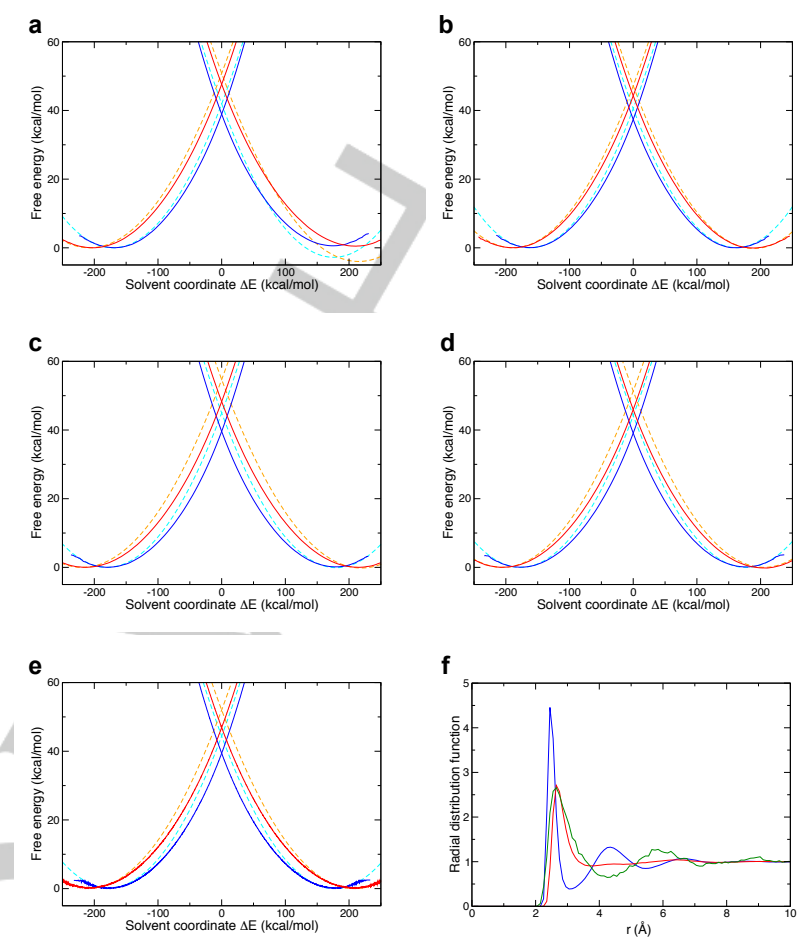

Figure 1. Diabatic free energy curves for the $A B$ pair at the interface in the perpendicular (a) and parallel geometries (b), in the middle of the slab in the perpendicular (c) and parallel geometries (d) and in the bulk (e) (see Scheme 1). Solid lines show the profiles obtained from a series of simulations biasing the solvent coordinate between its two equilibrium values for $R_{A B}=5 \AA$ (blue) and $R_{A B}=10 \AA$ (red). Dashes are the parabolic profiles obtained within the harmonic approximation from the equilibrium solvent coordinates (see e.g. ${ }^{[35,49]}$ ). f) Radial distribution function between anionic solute $A$ and water oxygen atoms (blue), cationic solute $B$ and water oxygen atoms (red), and between A and B (green) in a bulk aqueous solution.

\section{Equilibrium Reaction Free Energy $\Delta G_{r x n}$}

A first aspect of the preceding free energy curves to discuss is the equilibrium reaction free energy $\Delta \mathrm{G}_{\mathrm{rxn}}$.

In the bulk, in the middle of the slab and in the parallel arrangement at the interface, $\Delta \mathrm{G}_{\mathrm{rxn}}=0$ by symmetry. This contrasts with the situation at the interface with the perpendicular geometry (recall that $A$ remains in the interfacial region while $B$ moves away). The free energy profiles in Fig. 1a show that the ET reaction is slightly endergonic: $\Delta \mathrm{G}_{\mathrm{rxn}}=0.55 \mathrm{kcal} / \mathrm{mol}$ when $R_{A B}=5 \AA$, and $\Delta \mathrm{G}_{\mathrm{rxn}}=0.45 \mathrm{kcal} / \mathrm{mol}$ when $R_{A B}=10 \AA$ (note that the harmonic approximation incorrectly predicts a strongly exergonic ET reaction for both $R_{A B}$ distances; we return to that overestimate in our discussion of Fig. 4).

The key to understanding this free energy cost is the feature that the eq. 1 ET removes a negative charge from the surfaceadjacent $A$, replacing it with a positive charge. This strongly suggests that $\Delta \mathrm{G}_{\mathrm{r} \times \mathrm{n}}>0$ results from the greater stability of the anion in the interfacial region compared to that of the cation. This 
is confirmed in Fig. 2 which displays the solvation free energy profiles for the separate anion and cation as a function of distance $z$ from the graphene plate. By the same token, if the orientation of the reactant pair were reversed, with $B$ being the surface-adjacent member of the complex, the interfacial ET reaction becomes exergonic $\Delta G_{\mathrm{rxn}}<0$.

The propensity of ions to lie in the bulk or at a hydrophobic aqueous interface (including, e.g., the air/water interface) has been shown ${ }^{[50]}$ to be determined by the strength of the ion-water interaction (and by ion polarizability, not included in our simulations). The calculated solvent coordination numbers around the anion and cation solutes for different geometries and $R_{A B}$ separations (Fig. 3) provides a molecular understanding of the reason why the anion is more stable than the cation in the first layer next to the apolar graphene wall. The anionic solute strongly interacts with the water hydrogen atoms, which do not possess any Lennard-Jones sphere and can approach close to the anion. This explains the smaller cavity radius for the anion than for the cation (Fig 1.f) and the smaller coordination number for the anion than for the cation (Fig.3). When the solutes are moved from the middle of the slab to the interface, Fig. 3 shows that the decrease in coordination number is much more pronounced for the larger cation than for the smaller anion. The graphene wall-induced partial desolvation thus leads to a larger free energy cost for the cation than for the anion. The difference in solvation free energies for the cation and anion at the graphene interface $(\simeq 0.6 \mathrm{kcal} / \mathrm{mol})$ is in quantitative agreement with the $\Delta G_{\mathrm{rxn}}$ reaction free energy asymmetry.

\section{ET Solvent Reorganization Energy and Reaction Barrier}

In order to determine the impact of the interface and of the $A B$ pair orientation on the ET rate constant, we now turn to the solvent reorganization energy $\lambda$ and the closely related reaction barrier. We recall from the Introduction and section "Model electron transfer reaction" that $\lambda$ is key for the ET reaction rate via its barrier, which increases with increasing $\lambda$. We first determine $\lambda$ in each diabatic state from our simulated free energy profiles shown in Fig. 1 as

$$
\lambda_{\mathrm{i}}=\mathrm{G}_{\mathrm{i}}\left(\Delta E_{j}^{e q}\right)-\mathrm{G}_{\mathrm{i}}\left(\Delta E_{i}^{e q}\right)
$$

where $i, j=R, P$, and the $E T$ free energy barrier as

$$
\Delta \mathrm{G}^{\ddagger}=\mathrm{G}_{\mathrm{R}}(0)-\mathrm{G}_{\mathrm{R}}\left(\Delta \mathrm{E}_{\mathrm{R}}^{\mathrm{eq}}\right)
$$

We then compare these values with the harmonic approximation (sometimes referred to as "linear response") ${ }^{[49,51]}$

$$
\lambda_{\text {harm }}=\left(\Delta E_{P}^{e q}-\Delta E_{R}^{e q}\right) / 2
$$

and eq 2 for the free energy barrier in the harmonic approximation. The results in Fig 4 show that $\lambda$ and $\Delta G^{\ddagger}$ are very sensitive to the local environment (interface, middle of the slab, bulk) and to the $A B$ pair orientation: $\lambda$ and $\Delta G^{\ddagger}$ are smaller at the interface than in the middle of the slab, and this reduction is even more pronounced for the parallel geometry than for the perpendicular one.

The fairly large solvent reorganization energies in Fig. 4a arise from the model system's small solute radii and the resulting strong solute-solvent interaction; while these values are not meant to be realistic, this helps the simple harmonic approximation, which will facilitate our analysis (as shown in the SI, our conclusions are unchanged when a solute pair with weaker solute-solvent interaction is considered). In addition, the large reorganization energies $\lambda$ imply that when the $A-B$ complex is within the interfacial region, the $\Delta G_{\mathrm{rxn}}$ departure from a symmetric reaction (comparable to the thermal energy $k_{B} T$ and larger than this, though overestimated, in the harmonic approximation, see Fig. 1) has a small impact on the simulated reaction barrier height (Fig. $4 \mathrm{~b}$ ). A related consequence is that the Marcus inverse regime is not observed for this system.

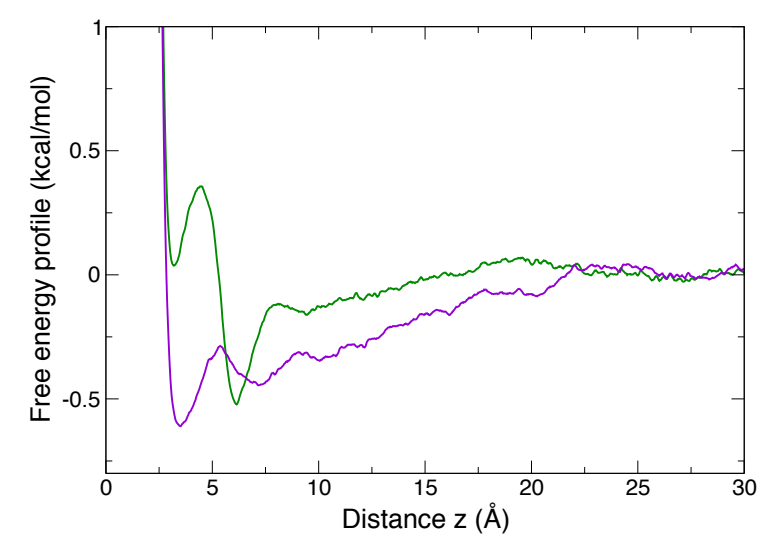

Figure 2. Solvation free energy profiles for the separate anion (violet) and cation (green) as a function of distance $z$ from the graphene plate.

a

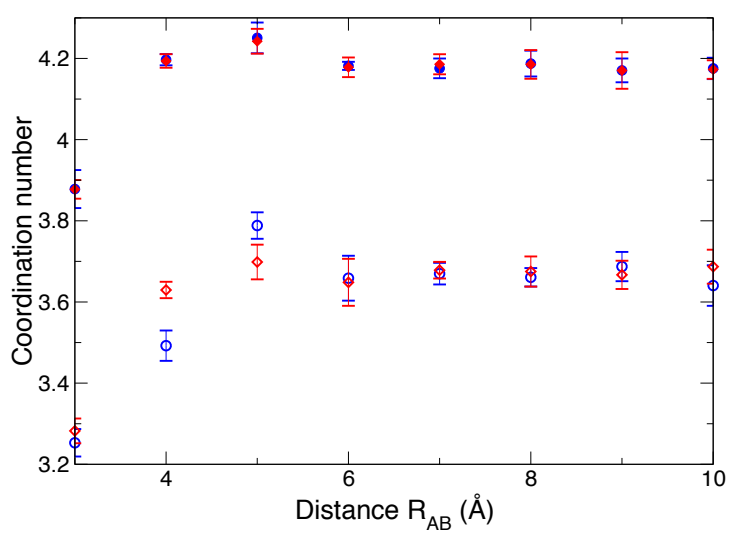

b

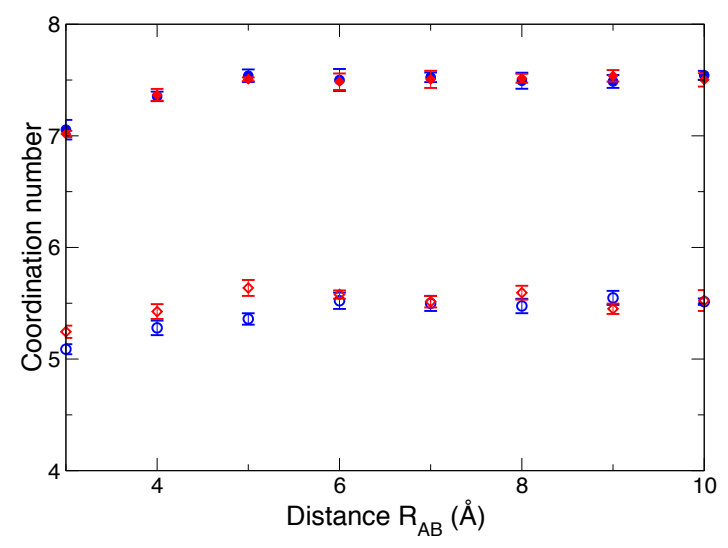


Figure 3. Coordination numbers, defined as the number of water oxygen atoms within the first shell around solute A-B's site A in its anionic reactant (a) and cationic product (b) state as a function of the $R_{A B}$ distance, respectively in the perpendicular (blue) and parallel (red) geometries (see Scheme 1) and where site A lies within the graphene interfacial layer (open symbols) and in the middle of the slab (full symbols).

Figure 4a shows that the harmonic approximation provides a good description of the changes in $\lambda$ between the interfacial, middle and bulk environments, and between the parallel and perpendicular orientations. One exception where the harmonic approximation deviates more markedly from the simulation results is the perpendicular geometry at the interface, due to the substantial difference between the $\lambda_{R}$ and $\lambda_{P}$ reorganization energies around the $\mathrm{R}$ and $\mathrm{P}$ states. As shown by the coordination numbers in Fig. 3 , this is caused by the contrast between the solvation structures in the first interfacial layer and in the further layers. The harmonic approximation also provides a good description of the reaction free energy barrier $\Delta \mathrm{G}^{\ddagger}$ (Fig. $4 \mathrm{~b}$ ) and its changes with the different environments and geometries. Its main limitation is its systematic but limited underestimation of the reaction's free energy, due to its underestimation of the solvent reorganization energy in the reactant state (Fig. 4a).

For simplicity, we adopt the harmonic approximation in the following, and we take advantage of its reduced computational cost compared to the full free energy profile calculation to examine how $\lambda$ changes over a broader range of locations for the reaction A-B complex. Figure 5 displays the harmonic approximation results eq 7 versus the separation $R_{A B}$ of the $A-B$ pair for the pairs' parallel and perpendicular orientation with respect to the graphene surface, successively for the complex with its A member always fixed in the first layer at the graphene interface, then for $A$ in the second layer, for the complex centered in the middle of the two graphene plates, and finally for the complex in the bulk.

In all cases, $\lambda$ increases with increasing $R_{A B}$. At smaller $R_{A B}$, the hydration shells of the anionic $A$ and cationic $B$ complex members interfere with each other to a degree. As $R_{A B}$ increases, $\lambda$ will increase, asymptotically approaching the higher cost of the reorganization of the separate, full hydration shells of the anionic and cationic components of the complex ${ }^{[52]}$. On the way to this limit, in the middle of the $6 \mathrm{~nm}$-thick slab, the orientation of the reaction complex's orientation matters less for $\lambda$ than at the interface; a residual anisotropy is nonetheless present, consistent with the long-range (approximately $3 \mathrm{~nm}$ ) decay length of the interface's effect on the dielectric constant along the direction perpendicular to the wall that was determined in our recent study [12]. On the other hand, in the interfacial geometries where the reorganization cost is lower, the parallel geometry $\lambda$ is noticeably lower than that for the perpendicular case. Finally, in the perpendicular geometry, moving $A$ from the first layer to the second layer next to the graphene wall leads to $\lambda$ values which are very close to those in the middle of the slab. We will return to these features presently.

\section{Dielectric Constant and Reorganization Energy}

We now determine the molecular origin of the $\lambda$ reduction at the interface, and examine the suggestion ${ }^{[13]}$ that it is due to the reduced local dielectric constant perpendicularly to the wall. Simulations have shown that the local dielectric constant at an interface is highly anisotropic: it is reduced relative to the bulk along the direction perpendicular to the wall, and typically enhanced along the parallel direction. This anisotropy is included in our simulations and is for example visible in the differences between the free energy profiles obtained with parallel and perpendicular orientations in Fig. 1. To analyze the changes in the solvent reorganization energy at the interface, we employ eq. 3. The latter was derived with a number of approximations, including a continuum solvent description and an isotropic environment; regarding the latter, its tensorial extension could be envisaged. Our focus is however on key aspects of the ET reaction rate. We therefore use eq. 3 and probe the system's anisotropy by examining $\lambda$ along the directions parallel and perpendicular to the wall and determining the impact of the dielectric constant anisotropy on the solvent reorganization energy. To this end, we consider the different solute pair orientations described in Scheme 1, and use the simple isotropic continuum model eq. 3 to probe the effective dielectric constant for each orientation.

We note that, remarkably, in all cases in Fig. 5, the A-B separation dependence of $\lambda$ found in the molecular simulations is very close to the $1 / R_{A B}$ dependence which follows from the dielectric continuum model eq. 3 (see also Fig. 6 below). In eq 3, the slope of $\lambda$ with $1 / R_{A B}$ only depends on $1 / \varepsilon$ (and on $\Delta q$ which is fixed in our model eq. 1). On the other hand, the intercept of $\lambda$ along $1 / R_{A B}$ depends both on $1 / \varepsilon$ and on the cavity radii $r_{A ; B}$ whose values cannot be unambiguously determined from the molecular simulations.

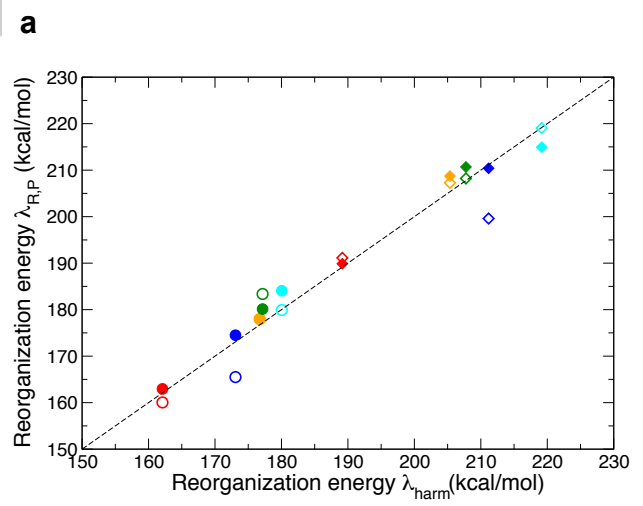

b

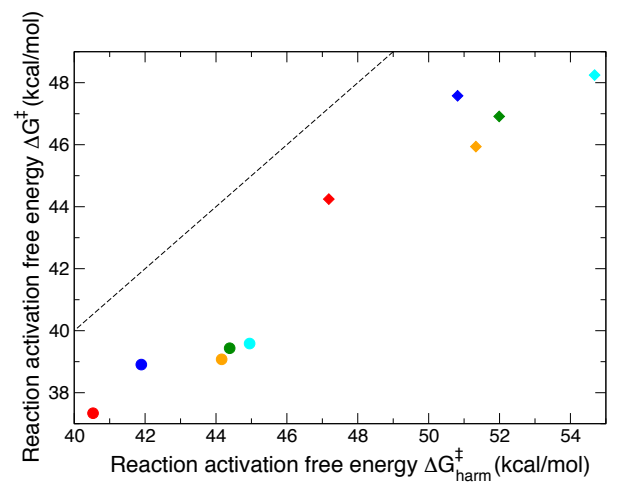

Figure 4. a) Reorganization energy from the free energy profiles (eq 5) as a function of the harmonic reorganization energy (eq 7), in the reactant (full symbols) and in the product (open symbols) diabatic states, at $R_{A B}$ distances of $5 \AA$ (circles) and $10 \AA$ (diamonds), in the bulk (green), at the interface in the perpendicular (blue) and parallel (red) geometries, and in the middle of the slab 
with the perpendicular (cyan) and parallel (orange) geometries (see Scheme 1). b) Reaction activation free energy from the free energy profiles as a function of the harmonic activation free energy (eq 2), with the same symbols as in panel

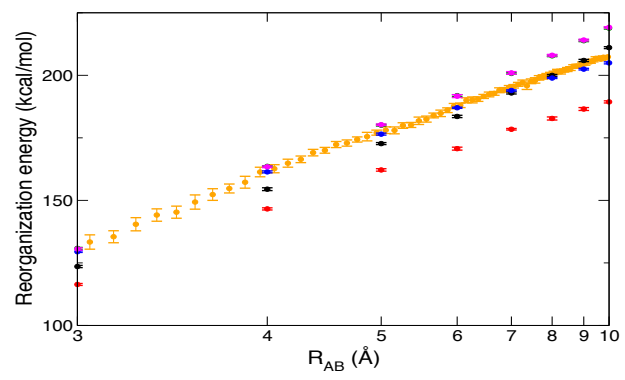

Figure 5. Reorganization energy (eq 7 ) as a function of the $R_{A B}$ distance plotted with a reciprocal scale for the horizontal axis, respectively in the perpendicular (black) and parallel (red) geometries at the interface, in the perpendicular (green) and parallel (blue) geometries in the middle of the slab, in the perpendicular geometry with $\mathrm{A}$ in the second layer next to the graphene sheet (pink) and in the bulk (orange) (see Scheme 1) (the pink and green symbols strongly overlap). The chosen 3-10 $\AA$ range for $R_{A B}$ extends from typical A-B distances for a contact ion pair (Fig. 1f) to long-range ET (limited in our simulations by the periodic simulation box size)

Given this slope dependence, one could try to determine an effective $\varepsilon$ from eq. 3 and the slope of $\lambda$ versus $1 / R_{A B}$. Unfortunately, due to the inverse proportionality in the dielectric constant, we find that the resulting uncertainty in $\varepsilon$ is too large to obtain meaningful $\varepsilon$ values.

We therefore adopt a different strategy. To analyze the dielectric constant aspects of the reorganization energy, we focus on the interfacial case, with the reaction complex member $A$ always in the interfacial layer, with member $B$ at various separations $R_{A B}$ from it and with the $A B$ direction either perpendicular or parallel to the graphene sheet. In particular, we compare the simulation results for $\lambda$ with the dielectric continuum formula eq. 3 , with three different choices for the description of the dielectric constant $\varepsilon$ : i) the bulk SPC/E dielectric constant $\varepsilon=71^{[53]}$, ii) the local interfacial dielectric constant $\varepsilon \simeq 2$ that was suggested ${ }^{[7,10]}$ from a 2 -state capacitor series interpretation of experimental and simulation results, assuming an extremely restricted first layer and a bulklike behavior in further layers ${ }^{[54]}$, and iii) the local dielectric constant profile that we recently determined ${ }^{[12]}$ from a Kirkwood factor analysis of molecular dynamics simulations. This last picture is sufficiently novel to require a brief description here. The dielectric constant along the direction perpendicular to the wall is reduced at the graphene interface due to the anisotropic dipolar field and the exclusion by the wall of water molecules that would bring a positive dipole-dipole correlation in the bulk. The dipoledipole interaction's long-range character induces a slow decay of the dielectric constant reduction on a scale of several nanometers from the wall. Due to this effect's strong anisotropy, the dielectric constant along the direction parallel to the wall is much less affected by the dipolar field anisotropy and excluded-volume combination; simulations showed that it is actually locally enhanced compared to the bulk ${ }^{[9,10,12,55]}(\varepsilon=175$ in the first layer $)$, due to the orientation of the first layer molecules which tend to lie with their dipole tangent to the wall (and only the first layer molecules are affected).
Figure $6 a$ shows, for the perpendicular $A B$ orientation, the $1 / R_{A B}$ dependence of $\lambda$ both from our simulations and from the dielectric continuum expression eq. 3 with the above-mentioned three different dielectric constant values (the intercept value is numerically adjusted via a linear regression). The $\varepsilon=2$ case leads to a serious underestimate of the slope and is not compatible with the simulation results. The bulk dielectric constant provides a better description, but the best agreement is reached with the local dielectric constant profile determined from our recent simulations ${ }^{[12]}$, and whose character was sketched above ${ }^{[56]}$.

In the opposite, parallel arrangement, Fig. $6 b$ shows again that the dielectric continuum expression with $\varepsilon=2$ cannot describe the $1 / R_{A B}$ dependence of $\lambda$. Both $\varepsilon=71$ and $\varepsilon=175$ (cf SI of ref ${ }^{[12]}$ ) provide satisfactory descriptions of the simulation results and the inverse dependence of the slope with the dielectric constant does not allow a more precise determination of its value (However, while the increased $\varepsilon=175$ value could be consistent with our calculated $1 / R_{A B}$ dependence of $\lambda$, it cannot explain the reduction in $\lambda$ at the interface, since eq. 3 shows that it would instead lead to an increase in $\lambda$ ).

The results in Fig 6 therefore show that the interfacial $\lambda$ is not consistent with a dramatically reduced dielectric constant at the interface, but that our recent analysis of the local dielectric constant provides an excellent description of the effective dielectric constant entering in the continuum model expression eq. 3.

Since we have concluded that the reduction in $\lambda$ at the interface is not due to an extremely low dielectric constant, we return to Fig. 5 to determine its molecular origin. Figure 5 shows that while the curves of $\lambda$ versus $1 / R_{A B}$ exhibit fairly similar slopes across the different environments and orientations, they are vertically shifted, i.e., they have different intercepts (Figures S2-3 show that a solute pair with a weaker solvent interaction yields very similar results). This indicates that the $\lambda$ reduction on passing from the bulk to the interface is mostly due to a change in the solvation of the $A B$ species. The decrease in coordination numbers shown in Fig. 3 for $A$ and $B$ upon movement from the middle of the slab to the interface reflects a partial desolvation due to the wall, which reduces the energy cost to change that solvation, i.e., the observed solvent reorganization energy decrease at the interface. This conclusion is further supported by the enhanced decrease in $\lambda$ for the parallel geometry, when both $A$ and $B$ are partly desolvated in the first layer. This stresses the importance of the interfacial solvation shell structure that has been recognized in other contexts ${ }^{[21,26,30,33]}$. As reviewed e.g. in ref. [57], this highlights an important limitation of dielectric continuum models which do not correctly describe these hydration structure changes at interfaces. Finally, the considerations of the present work should be relevant for a number of interfacial elementary chemical processes, including not only ET reactions but also proton transfer reactions ${ }^{[58]}$ and solvation dynamics ${ }^{[59]}$ for which reduced solvent polarity at an interface has been shown to have an important impact. 


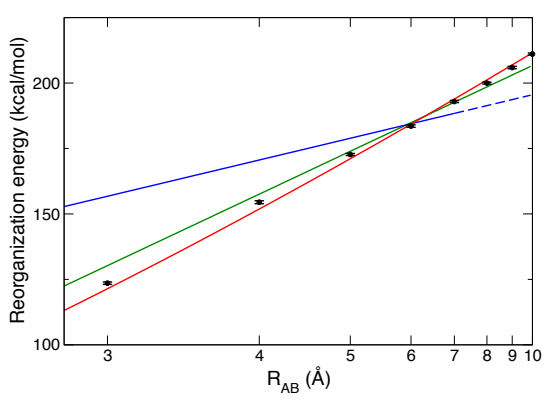

b

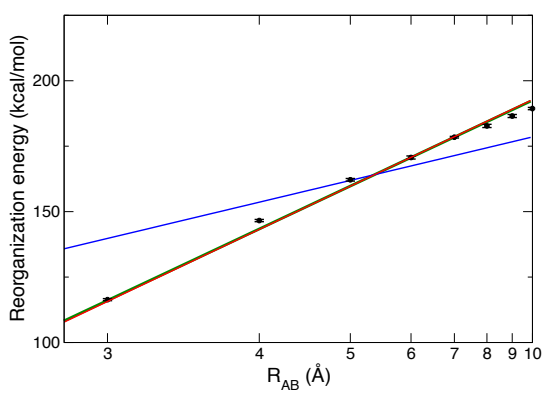

Figure 6. a) Reorganization energy (eq 6) in the perpendicular geometry at the interface as a function of the $R_{A B}$ distance plotted with a reciprocal scale for the horizontal axis from our simulations (black symbols) and fits using the continuum equation 3 with $\varepsilon=71^{[53]}$ (green), $\varepsilon=2$ (blue) and the dielectric constant profile $\varepsilon_{\perp}(\mathrm{z})$ determined in ref. ${ }^{[12]}(\mathrm{red})$; the $\varepsilon=2$ curve is extended with dashes when $B$ moves beyond the suggested ${ }^{[7]}$ restricted interfacial layer thickness. b) Idem in the parallel geometry, where the red curve is the enhanced $\varepsilon=175$ parallel interfacial dielectric constant determined in ref. ${ }^{[12]}$, and the green curve $\varepsilon=71$ overlaps with the red curve.

\section{Concluding Remarks}

Here we have examined, by simulations and extensive analysis, a model electron transfer reaction within liquid water confined between two graphene sheets. The chosen model system's simplicity allows our focus on solvation and solvent dielectric constant effects for the reaction without the influence of other obscuring features. Our molecular dynamics simulations are analyzed with the Marcus model dielectric continuum approximation for $\lambda$ and the system's anisotropy is probed by considering parallel and perpendicular orientations of the reactive pair relative to the wall. Our principal conclusion is that a reduction of the reorganization energy and the reaction's activation free energy barrier in the interfacial region is not due to a muchreduced dielectric constant perpendicularly to the graphene sheet but rather to a wall-induced effect of desolvation of the reaction complex. Future efforts will address improved realistic models of the reaction, assessment of dynamical effects on the rate ${ }^{[38]}$, and most especially, corresponding considerations of electrochemical reactions involving electron transfer to and from an electrode.

Keywords: Electron transfer reaction $\cdot$ Confinement $\cdot$ Dielectric constant $\cdot$ Solvent reorganization energy $•$ Aqueous solution

[1] J. C. Rasaiah, S. Garde, G. Hummer, Annu Rev Phys Chem 2008, 59, 713-740.

[2] M. D. Fayer, N. E. Levinger, Annu Rev Anal Chem 2010, 3, 89-107.

[3] W. H. Thompson, Annu Rev Phys Chem 2011, 62, 599-619.
H. Li, J. Xiao, Q. Fu, X. Bao, Proc Natl Acad Sci U S A 2017, 114, 5930-5934.

[5] M. Seo, T. D. Chung, Curr Opin Electrochem 2019, 13, 47-54.

[6] O. Teschke, G. Ceotto, E. F. de Souza, Phys Rev E 2001, 64, 011605.

[7] L. Fumagalli, A. Esfandiar, R. Fabregas, S. Hu, P. Ares, A Janardanan, Q. Yang, B. Radha, T. Taniguchi, K. Watanabe, G. Gomila, K. S. Novoselov, A. K. Geim, Science 2018, 360, 1339-1342.

[8] M. H. Motevaselian, N. R. Aluru, ACS Nano 2020, 14, 12761-12770.

[9] S. Ruiz-Barragan, D. Muñoz-Santiburcio, S. Körning, D. Marx, Phys Chem Chem Phys 2020, 22, 10833-10837.

[10] P. Loche, C. Ayaz, A. Wolde-Kidan, A. Schlaich, R. R. Netz, J Phys Chem B 2020, 124, 4365-4371.

[11] S. Mondal, B. Bagchi, Nano Letters 2020, 20, 8959-8964.

[12] J.-F. Olivieri, J. T. Hynes, D. Laage, J Phys Chem Lett 2021, 12 4319-4326.

[13] R. E. Bangle, J. Schneider, D. T. Conroy, B. M. Aramburu-Trošelj, G. J. Meyer, J Am Chem Soc 2020, 142, 14940-14946.

[14] A. Ambrosi, C. K. Chua, N. M. Latiff, A. H. Loo, C. H. A. Wong, A. Y.

S. Eng, A. Bonanni, M. Pumera, Chem Soc Rev 2016, 45, 2458-2493.

A. Kaplan, Z. Yuan, J. D. Benck, A. Govind Rajan, X. S. Chu, Q. H.

Wang, M. S. Strano, Chem Soc Rev 2017, 46, 4530-4571.

[16] G. Gnana kumar, G. Amala, S. M. Gowtham, RSC Advances 2017, 7, 36949-36976.

[17] P. Liu, T. Yan, L. Shi, H. S. Park, X. Chen, Z. Zhao, D. Zhang, J Mater Chem A 2017, 5, 13907-13943.

[18] Y. Zhang, G. Stirnemann, J. T. Hynes, D. Laage, Phys Chem Chem Phys 2020, 22, $10581-10591$.

[19] Y. Zhang, H. B. de Aguiar, J. T. Hynes, D. Laage, J Phys Chem Lett 2020, 11, 624-631.

[20] J. B. Straus, G. A. Voth, J Phys Chem 1993, 97, 7388-7391.

[21] X. Xia, M. L. Berkowitz, Chem Phys Lett 1994, 227, 561-566.

[22] D. A. Rose, I. Benjamin, J Chem Phys 1994, 100, 3545-3555.

D. A. Rose, I. Benjamin, Chem Phys Lett 1995, 234, 209-215.

[24] Y.-P. Liu, M. D. Newton, J Phys Chem 1994, 98, 7162-7169.
[25] J. B. Straus, A. Calhoun, G. A. Voth, J Chem Phys 1995, 102, 529 539.

[26] C. Hartnig, M. T. Koper, J Am Chem Soc 2003, 125, 9840-9845.

[27] S. K. Reed, P. A. Madden, A. Papadopoulos, J Chem Phys 2008 , 128, 124701.

[28] A. P. Willard, S. K. Reed, P. A. Madden, D. Chandler, Faraday Discuss. 2009, 141, 423-441.

[29] R. C. Remsing, I. G. McKendry, D. R. Strongin, M. L. Klein, M. J. Zdilla, J Phys Chem Lett 2015, 6, 4804-4808.

[30] Z. Li, G. Jeanmairet, T. Méndez-Morales, M. Burbano, M. Haefele, M. Salanne, J Phys Chem Lett 2017, 8, 1925-1931.

[31] R. R. Nazmutdinov, M. D. Bronshtein, E. Santos, J Phys Chem C 2019, 123, 12346-12354.

A. M. Limaye, W. Ding, A. P. Willard, J Chem Phys 2020, 152, 114706

[33] S. A. Shermukhamedov, R. R. Nazmutdinov, M. D. Bronshtein, M. Probst, ChemElectroChem 2021, 8, 563-569.

R. A. Marcus, J Chem Phys 1956, 24, 966.

M. D. Newton, Adv Chem Phys 1999, 106, 303-375

[36] M. Newton, in Electron transfer in chemistry (Eds.: V. Balzani.), Wiley$\mathrm{VCH}$, Weinheim, 2001, pp. 3-63.

[37] J.-M. Savéant, C. Costentin. Elements of molecular and biomolecular electrochemistry; Wiley: New Jersey, 2006;

[38] D. A. Zichi, G. Ciccotti, J. T. Hynes, M. Ferrario, J Phys Chem 1989 , 93, 6261-6265.

[39] M. B. Robin, P. Day, Adv. Inorg. Chem. Radiochem. 1968, 10, $247-$ 422.

[40] M. Born, Z Phys 1920, 1, 45-48.

[41] B. Roux, H. A. Yu, M. Karplus, J Phys Chem 1990, 94, 4683-4688.

[42] A. A. Rashin, B. Honig, J Phys Chem 1985, 89, 5588-5593.

[43] G. Hummer, L. R. Pratt, A. E. Garcia, J Phys Chem 1996, 100, 12061215.

[44] M. K. Rana, A. Chandra, J Chem Phys 2013, 138, 204702.

[45] I.-C. Yeh, M. L. Berkowitz, J Chem Phys 1999, 111, 3155-3162.

[46] S. Plimpton, J Comp Phys 1995, 117, 1-19.

[47] G. Bussi, D. Donadio, M. Parrinello, J Chem Phys 2007, 126, 014101

[48] M. Souaille, B. Roux, Comput Phys Commun 2001, 135, 40-57.

J. Blumberger, Chem Rev 2015, 115, 11191-11238

[50] P. Jungwirth, D. J. Tobias, Chem Rev 2006, 106, 1259-1281.

[51] D. W. Small, D. V. Matyushov, G. A. Voth, J Am Chem Soc 2003 125, 7470-7478

[52] M. Tachiya, J Phys Chem 1993, 97, 5911-5916.

[53] M. R. Reddy, M. Berkowitz, Chem Phys Lett 1989, 155, 173-176.

[54] While $\varepsilon=2$ is the continuum value for a solely electronically polarizable liquid, our molecular dynamics water solvent is not. We select the value 2 as a representative very low $\varepsilon$ value; our qualitative conclusions would also hold for the choice $\varepsilon=1$.

[55] M. H. Motevaselian, N. R. Aluru, J Phys Chem Lett 2020, 11, 1053210537. 
[56] However, we note that for considerably narrower slabs, the dielectric constant at the interface is expected to decrease, as explained in our recent work [12].

[57] I. Benjamin, Annu Rev Phys Chem 2015, 66, 165-188.

[58] K. R. Mitchell-Koch, W. H. Thompson, J Phys Chem B 2008, 112

7448-7459.

[59] W. H. Thompson, J Chem Phys 2004, 120, 8125-8133. 


\section{Entry for the Table of Contents}

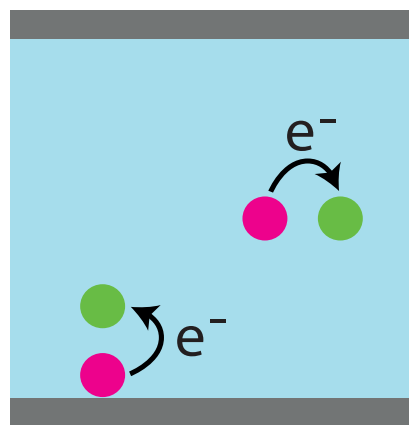

We have studied, via simulation and analysis, the reorganization energy and free energy reaction barrier for a model electron transfer reaction occurring at various locations within liquid water confined between two graphene sheets. We find that the acceleration of the reaction in the interfacial region by reduction of both these quantities is due not to significant local dielectric constant reduction, but to desolvation of the reaction complex.

Institute and/or researcher Twitter usernames:

@Chimie_ENS

@INC_CNRS 


\title{
Supplementary Information
}

\section{A Model Electron Transfer Reaction in Confined Aqueous Solution}

\author{
Jean-François Olivieri, ${ }^{[a]}$ Damien Laage ${ }^{*[a]}$ and James T. Hynes ${ }^{\star[a, b]}$
}

[a] PASTEUR, Department of Chemistry, École Normale Supérieure, PSL University, Sorbonne Université, CNRS 75005 Paris, France

E-mail: damien.laage@ens.psl.eu

[b] Department of Chemistry, University of Colorado

Boulder, CO 80309, USA

E-mail: casey.hynes@ens.psl.eu

\section{Simulation Methodology: Combination Rules}

For the carbon-oxygen and carbon-solute Lennard-Jones (LJ) interaction potentials, we followed the approach of ref. ${ }^{[1]}$ where carbon-oxygen LJ parameters were optimized to reproduce DFT-based molecular dynamics simulation results. A modified LJ potential was introduced where the attractive term was scaled

$$
V_{i j}\left(r_{i j}\right)=4 \varepsilon_{i j}\left[\left(\frac{\sigma_{i j}}{r_{i j}}\right)^{12}-\lambda\left(\frac{\sigma_{i j}}{r_{i j}}\right)^{6}\right] .
$$

and Lorentz-Berthelot mixing rules were used to determine the cross parameters for the interaction between atoms $i$ and $j, \sigma_{i j}=\left(\sigma_{i i}+\sigma_{j j}\right) / 2$ and $\epsilon_{i j}=\sqrt{\epsilon_{i i} \epsilon_{j j}}$. The optimized values for the graphene carbon atoms were determined in ref. ${ }^{[1]}$ to be $\varepsilon_{C c}=0.3598 \mathrm{~kJ} / \mathrm{mol}$ and $\sigma_{c c}=3.4 \AA$, and the same tuning parameter $\lambda=0.7$ was used for the carbon-oxygen and carbonsolute LJ interactions. Solute-oxygen and solute-water LJ interactions employed the Lorentz-Berthelot mixing rules with no scaling.

\section{Density Profiles}

Figure $\mathrm{S} 1$ shows the water oxygen and hydrogen number density profiles across the slab, together with a typical configuration of the A-B pair in configuration $i$ (see Scheme 1) with $A$ in the first layer and the $A B$ direction perpendicular to the interface. The relative sizes of the $A$ and $B$ solutes with respect to the hydration layers' thickness are rendered by the colored disks, whose diameters are the Lennard-Jones $\sigma_{A}$ and $\sigma_{B}$.

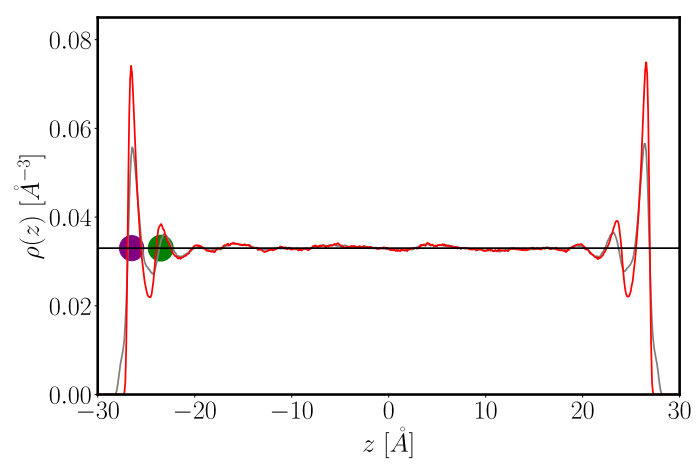

Figure S1. Water oxygen (red) and hydrogen (gray, scaled by $1 / 2$ ) number density profiles across the water slab, and positions of the A and B sites in the interfacial perpendicular arrangement ( $i$ in Scheme 1) for $R_{A B}=4 \AA$.

\section{Solute Size Effect}

We repeated the solvent reorganization energy calculations for larger $A$ and $B$ solutes $\left(\sigma_{A}=\sigma_{B}=3.5 \AA\right)$, which lead to weaker solute-solvent interaction energies and smaller solvent reorganization energies. Figures S2 and S3 show that the 
results for these weaker solute-solvent interaction energies exhibit the same behavior for the solvent reorganization energy dependence on the $R_{A B}$ distance as reported in the main text in Figures 5 and 6.

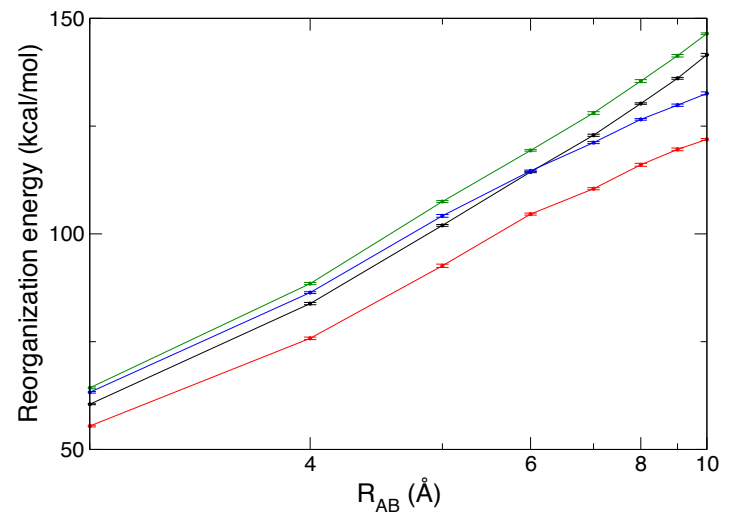

Figure S2. Analog of Figure 5, here for larger $A$ and $B$ solutes $\left(\sigma_{A}=\sigma_{B}=3.5 \AA\right)$. Reorganization energy (eq 7$)$ as a function of the $R_{A B}$ distance plotted with a reciprocal scale for the horizontal axis, respectively in the perpendicular (black) and parallel (red) geometries at the interface, in the perpendicular (green) and parallel (blue) geometries in the middle of the slab.

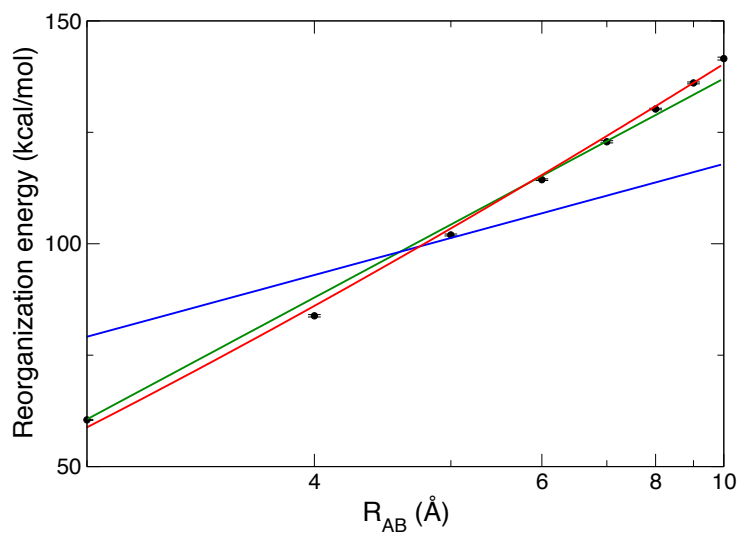

Figure S3. Analog of Figure $6 a$, here for larger $A$ and $B$ solutes $\left(\sigma_{A}=\sigma_{B}=3.5 \AA\right.$ ). Reorganization energy (eq 6$)$ in the perpendicular geometry at the interface as a function of the $R_{A B}$ distance plotted with a reciprocal scale for the horizontal axis from our simulations (black symbols) and fits using the continuum equation 3 with $\varepsilon=71^{[2]}$ (green), $\varepsilon=2$ (blue) and the dielectric constant profile $\varepsilon_{\perp}(\mathrm{z})$ determined in ref. [3] (red). A fit with the enhanced $\varepsilon=175$ parallel interfacial dielectric constant determined in ref. [3] is indistinguishable from the $\varepsilon=71$ fit (green).

M. K. Rana, A. Chandra, J Chem Phys 2013, 138, 204702.

M. R. Reddy, M. Berkowitz, Chem Phys Lett 1989, 155, 173-176.

[3] J.-F. Olivieri, J. T. Hynes, D. Laage, J Phys Chem Lett 2021, 12, 4319-4326. 